\title{
Pulmonary vascular enlargement and lesion extent on computed tomography are correlated with COVID-19 disease severity
}

\author{
Ryo Aoki $^{1,2} \cdot$ Tae Iwasawa $^{1,2}$ (1) $\cdot$ Eri Hagiwara $^{3} \cdot$ Shigeru Komatsu $^{3} \cdot$ Daisuke Utsunomiya $^{1,2} \cdot$ Takashi Ogura $^{3}$
}

Received: 26 November 2020 / Accepted: 23 December 2020 / Published online: 27 January 2021

(c) Japan Radiological Society 2021

\begin{abstract}
Purpose To assess the relationships among pulmonary vascular enlargement, computed tomography (CT) findings quantified with software, and coronavirus disease (COVID-19) severity.

Materials and methods Ultra-high-resolution (UHR) CT images of 87 patients (50 males, 37 females; median age, 63 years) with COVID-19 confirmed using real-time polymerase chain reaction were analyzed. The maximum subsegmental vascular diameter was measured on CT. Total CT lung volume (CTLV total) and lesion extent (ratio of lesion volume to CTLV total) of ground-glass opacities, reticulation, and consolidation were measured using software. Maximum pulmonary vascular diameter and lesion extent were analyzed using Spearman's correlation analysis. Logistic regression analysis was performed on CT results to predict disease severity. We also assessed changes in these measures on follow-up scans in 16 patients.

Results All 23 patients with severe and critical illness had vascular enlargement ( $>4 \mathrm{~mm})$. Pulmonary vascular enlargement (odds ratio 3.05, $p=0.018$ ) and CT lesion extent (odds ratio 1.07, $p=0.002$ ) were independent predictors of disease severity after adjustment for age and comorbidities. On follow-up CT, vascular diameter and CT lesion volume decreased $(p=0.001$, $p=0.002$; respectively), but CTLV total did not change significantly.

Conclusion Subsegmental vascular enlargement is a notable finding to predict acute COVID-19 disease severity.
\end{abstract}

Keywords COVID-19 $\cdot$ Lung $\cdot$ Computed tomography $\cdot$ Pneumonia $\cdot$ Computer-aided diagnosis

\section{Introduction}

As of November 17, 2020, more than 53.7 million cases of coronavirus disease 2019 (COVID-19)-a disease caused by severe acute respiratory syndrome coronavirus

Supplementary Information The online version contains supplementary material available at https://doi.org/10.1007/s1160 4-020-01085-2.

Tae Iwasawa

tae_i_md@wb3.so-net.ne.jp

1 Department of Radiology, Kanagawa Cardiovascular and Respiratory Center, 6-16-1 Tomioka-higashi, Kanazawa-ku, Yokohama, Kanagawa 236-8651, Japan

2 Department of Diagnostic Radiology, School of Medicine, Yokohama City University, 3 Chome-9 Fukuura, Kanazawa-ku, Yokohama, Kanagawa 236-0004, Japan

3 Department of Respiratory Medicine, Kanagawa Cardiovascular and Respiratory Center, 6-16-1 Tomioka-higashi, Kanazawa-ku, Yokohama, Kanagawa 236-8651, Japan
2 (SARS-CoV-2) infection-have been reported globally, including over 1.3 million deaths [1]. Hypoxic respiratory failure is a critical issue in COVID-19, and acute respiratory distress syndrome (ARDS) with diffuse alveolar damage is a common pulmonary lesion in COVID-19 [2]. Endothelial injury and disrupted vasoregulation have been proposed as key components in early COVID-19-related ARDS [3, 4]. A previous study using dual-energy computed tomography (CT) has demonstrated perfusion abnormalities in COVID19 , supporting this hypothesis $[5,6]$. However, the accessibility of dual-energy CT remains limited, and contrast material is not routinely used in patients with COVID-19.

Vascular abnormalities in the lung on non-contrast enhanced CT have been reported [7-10]. Caruso reported "subsegmental vessel enlargement" as an imaging feature on non-contrast-enhanced CT of patients with COVID-19 [9]. Wang reported a "vascular thickening sign" [10], which is the enlarged peripheral vessels in CT lesions. These reports showed that the vascular abnormalities were related with visual score on CT. However, the relationship between vessel enlargement and computer-based quantitative results of 
other CT findings, such as extent of ground-glass opacities (GGOs) and consolidation in the lung, has not been fully investigated.

In this study, we measured the subsegmental vessel diameter and CT lesion volume using ultra-high-resolution CT images (UHR-CT) to assess the relationship between CT findings quantified with software and COVID-19 disease severity. We also evaluated changes in these quantitative CT findings on follow-up CT scans obtained more than 1 month after disease onset.

\section{Materials and methods}

\section{Patient population}

Our institutional review board approved of this retrospective, single-center study and waived the requirement for obtaining informed patient consent (KCRC-20-0008). Consecutive hospitalized patients diagnosed with COVID-19 who underwent initial UHR-CT at our institution between February 12 and August 31, 2020 were enrolled. Throat swabs of all patients were tested for COVID-19 using real-time polymerase chain reaction tests. Patients who were moved to our hospital from another hospital after treatment with a respirator $(n=1)$ and those unable to perform appropriate breath-hold for CT $(n=4)$ were excluded. We also evaluated the follow-up CT images obtained more than 30 days after the initial CT scans in 16 patients. A final total of 87 patients and $103 \mathrm{CT}$ examinations were included in the study.

Clinical characteristics were collected from medical records, including age, sex, smoking history, presence of comorbidities, date of laboratory admission, laboratory findings [white blood cell (WBC) count, lymphocyte count, C-reactive protein (CRP), lactate dehydrogenase (LDH), brain natriuretic peptide (BNP), and D-dimer levels], and time from initial symptom onset to baseline CT.

\section{CT data acquisition}

All baseline CT images were obtained using a UHR-CT scanner (Aquilion Precision, Canon Medical Systems, Otawara, Japan) at full inspiration, with a tube voltage of 120 $\mathrm{kVp}$ with automatic tube current modulation. The median effective dose (interquartile range) of the initial UHR-CTs was $6.87(6.10-8.00) \mathrm{mSv}$. These data were calculated based on the dose-length products $(\mathrm{mGy} \bullet \mathrm{cm})$ and a k-factor of $0.140\left(\mathrm{mSv} \bullet \mathrm{mGy}^{-1} \bullet \mathrm{cm}^{-1}\right)$ [11]. UHR-CT images were reconstructed with two patterns: (1) high resolution mode $(1024 \times 1024$ matrix size, 0.25 -mm slice thickness, and deep learning reconstruction algorithm), which was used for visual evaluation by radiologists, and (2) conventional mode $(512 \times 512$ matrix size, 0.5 - $\mathrm{mm}$ slice thickness, and iterative reconstruction algorithm for mediastinum) (AIDR3D, FC03), which was used for computer-based quantification. All follow-up CT scans except two were also obtained using UHR-CT. Two follow-up CT scans were acquired using 320row multidetector CT (Aquilion ONE GENESIS, Canon Medical Systems) and reconstructed only with the conventional mode.

\section{Quantitative evaluation}

Two radiologists with 5-year and 29-year experience in chest CT imaging independently measured the diameter of several subsegmental vessels near CT lesions, which were close to the subsegmental bronchi (Fig. 1), using workstation calipers (Ziostation 2, Ziosoft, Tokyo, Japan), and the maximum vessel diameter of patients was recorded according to a technique used in a previous study [9]. The diameter was measured on axial and multiplanar reconstructed images. Vessel diameters were not measured in consolidations. When we did not find CT lesions such as GGOs, reticulation, or consolidation, we measured vessel diameters in the right lower lobe. The agreement between the diameters measured by the two radiologists was evaluated using intraclass correlation coefficients. We used the mean of the two radiologists' results as the maximum subsegmental vascular diameter of the patient.

Total CT lung volume (CTLVtotal) and lesion volume were measured using Quantification software (Quantification by Ziosoft Informatics Platform for interstitial lung disease, QZIP, Ziosoft), which is an automatic measurement system that uses a deep learning-based algorithm (Fig. 1, and Table E1 in the supplement material). The CT lesion extent was calculated as the ratio of lesion volume to CTLVtotal.

\section{Statistical analysis}

Disease severity was evaluated according to the COVID19 treatment guidelines [12] as follows: (1) mild-moderate condition (asymptomatic patients and patients with mild and moderate illness without oxygen therapy), and (2) severe-critical condition (patients with severe and critical illness with oxygen saturation $<94 \%$ and those requiring oxygen therapy during hospitalization). Binary logistic regression analysis was performed to determine whether baseline CT results were associated with the severe-critical condition. Variable of $p$ values less than 0.05 by univariate logistic regression analysis were chosen as the variables for multivariate logistic regression analysis. The correlation among maximum subsegmental vascular diameter, lesion volume in baseline CT images, and laboratory data were analyzed using Spearman's correlation coefficient. We used Wilcoxon's signed-rank test to compare between baseline and follow-up CT results. 

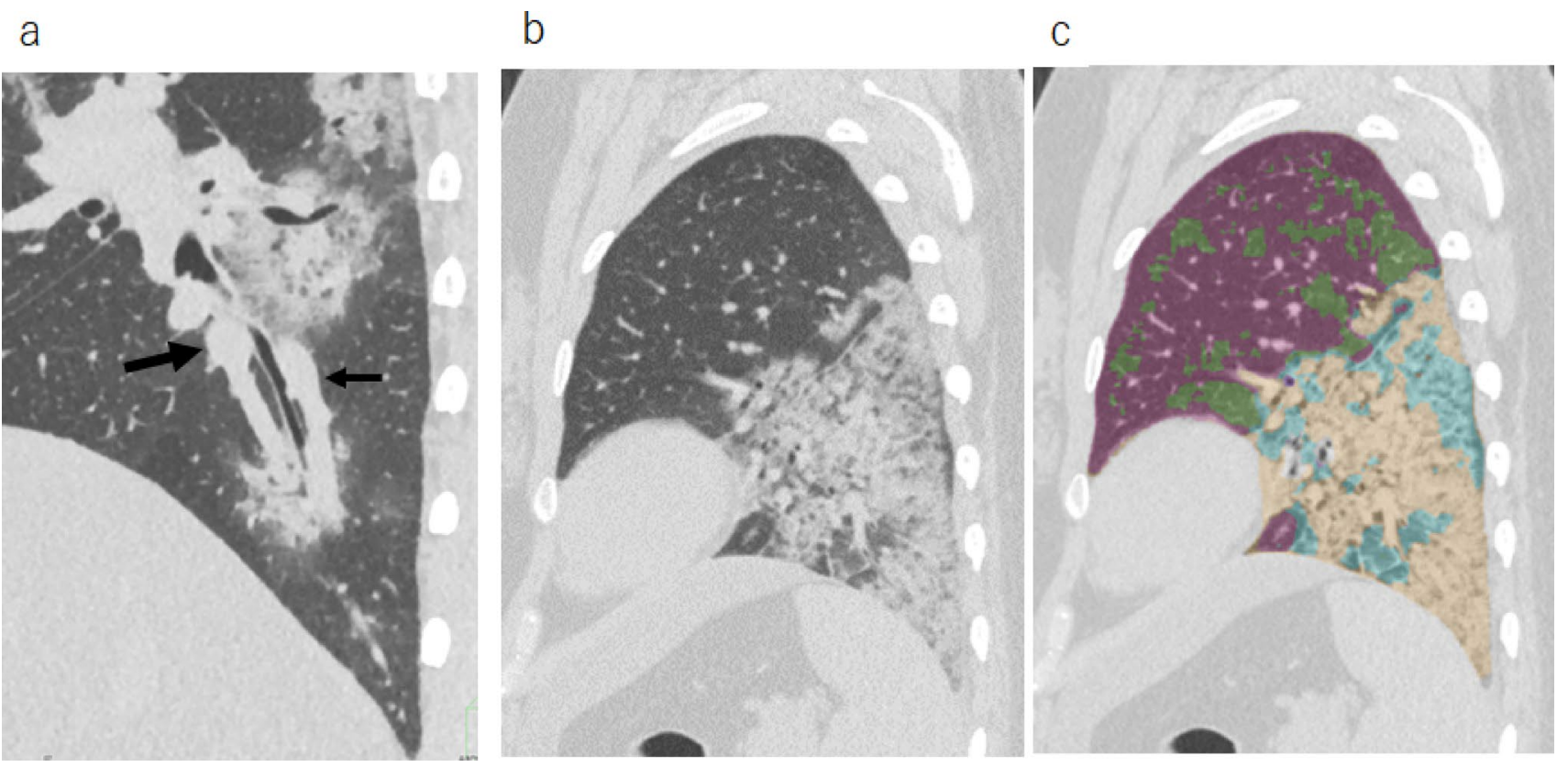

Fig. 1 CT images of a 37-year-old female with dyspnea. a Sagittal reconstruction image of ultra-high-resolution CT on day 7 from symptom onset showed dilated vessels close to the subsegmental bronchi. The large arrow indicates the pulmonary artery, and the small arrow indicates the pulmonary vein. b Sagittal image

All numeric data are reported as medians with quartiles. SPSS version 26 (IBM, Armonk, NY) was used for data analysis. A $p$ value $<0.05$ was considered statistically significant.

\section{Results}

Table 1 shows the clinical parameters and CT quantification data of all 87 patients, comprising 50 males and 37 females with a median age of 63 years (range 21-84 years). Of 87 patients, 23 required oxygen therapy, of whom two died due to respiratory failure. The median interval of symptom onset and baseline CT was 6 days (range 0-16 days). No abnormalities were detected on CT images in nine patients, whereas GGOs, reticulation, and consolidation were observed in the remaining 78 patients.

Table 1 shows the quantitative CT results. The intraclass correlation coefficient of the maximum subsegmental vascular diameter was 0.813 [95\% confidence interval (CI) $0.728-0.874]$ between the two radiologists. The median maximum subsegmental vascular diameter was $4.75 \mathrm{~mm}$. Subsegmental vascular enlargement (maximum subsegmental diameter $>4 \mathrm{~mm}$ ) was seen in 66 patients $(75.9 \%)$ and in all patients with the severe-critical condition (Fig. 2). Notably, four patients with severe-critical disease showed and c computer-based segmentation image; violet $=$ normal; light green $=\mathrm{GGO}$; beige $=$ consolidation; light blue $=$ reticulation. In this case, the sum of the CT lesion extent was $30.2 \%$. CT computed tomography, $G G O$ ground-glass opacity

vascular enlargement ( $>4 \mathrm{~mm}$ ), but they had a small amount of CT lesions $(<10 \%)$ (Figs. 2, 3c).

Table 2 shows the correlations among CT results and laboratory data. Interestingly, CTLVtotal decreased with a decrease in normal CT lung volume $(r=0.891, p<0.001)$ and an increase in the absolute volume of consolidation $(r=-0.344, p=0.001)$ (Fig. 3). CT lesion extent was positively correlated with oxygen saturation, CRP, LDH, and D-dimer levels. Maximum subsegmental vascular diameter was found to be correlated with CRP $(r=0.484, p<0.001)$ and oxygen saturation $(r=-0.406, p<0.001)$ levels, and positively correlated with CT lesion extent of GGOs $(r=0.355, p=0.001)$, reticulation $(r=0.475, p<0.001)$, and consolidation $(r=0.473, p<0.001)$. However, BNP level was not significantly correlated with vascular diameter and CT lesion extent of GGOs, reticulation, and consolidation.

Univariate logistic analysis showed that older age (odds ratio: $1.05 ; 95 \%$ CI $1.01-1.10, p=0.008)$, presence of comorbidities (odds ratio: 3.6; 95\% CI 1.19-10.9, $p=0.023$ ), elevated CRP level (odds ratio: 1.28; 95\% CI 1.11-1.48, $p=0.001$ ), and elevated LDH level (odds ratio: $1.01 ; 95 \% \mathrm{CI}$ $1.01-1.02, p=0.001$ ) were associated with the severe-critical condition. It also revealed that subsegmental vessel enlargement was associated with severe illness (odds ratio: $3.25 ; 95 \%$ CI 1.63-6.49, $p=0.001$ ). A large CT lesion extent was also significantly associated with the severe-critical condition. The odds ratios were 1.08 (95\% CI 1.02-1.15, 
Table 1 Patient clinical characteristics

\begin{tabular}{|c|c|c|c|c|}
\hline \multirow[t]{2}{*}{ Demographics and clinical characteristics } & & \multirow[t]{2}{*}{ Number (\%), or median (IQR) } & \multicolumn{2}{|l|}{ Univariate analysis } \\
\hline & & & Odds ratio and $95 \% \mathrm{CI}$ & $p$ value \\
\hline \multicolumn{2}{|l|}{ Total number of patients } & \multicolumn{3}{|l|}{87} \\
\hline \multicolumn{2}{|c|}{ Illness severity (asymptomatic, mild, moderate, severe, and critical) } & \multicolumn{3}{|l|}{$5 / 4 / 55 / 19 / 4(5.7 / 4.6 / 63.2 / 21.8 / 4.6 \%)$} \\
\hline \multicolumn{2}{|l|}{$\mathrm{SpO}_{2}$ during $\mathrm{CT}(\%)$} & \multicolumn{3}{|l|}{$96(94,97)$} \\
\hline \multicolumn{2}{|l|}{ Age (years) } & $63(49,71)$ & $1.05(1.01-1.10)$ & $0.008 *$ \\
\hline \multicolumn{2}{|l|}{$\operatorname{Sex}(M / F)$} & $50 / 37(57.4 / 42.5 \%)$ & $1.70(0.65-4.44)$ & 0.278 \\
\hline \multicolumn{2}{|l|}{ Smoking history (yes) } & $47(54.0 \%)$ & $2.27(0.86-6.03)$ & 0.099 \\
\hline \multicolumn{2}{|l|}{ Presence of comorbidity } & $50(57.4 \%)$ & $3.6(1.19-10.9)$ & $0.023 *$ \\
\hline \multicolumn{5}{|l|}{ Laboratory tests on admission } \\
\hline \multicolumn{2}{|l|}{ White blood cell count (cell/ $\mu \mathrm{L})$} & $5180(3880,6610)$ & $1.0(1-1.001)$ & 0.111 \\
\hline \multicolumn{2}{|l|}{ Lymphocyte count (cell/ $\mu \mathrm{L}$ ) } & $117(663,1484)$ & $0.999(0.997-1.0)$ & 0.052 \\
\hline \multicolumn{2}{|l|}{ C-reactive protein $(\mathrm{mg} / \mathrm{dL})$} & $2.56(0.57,6.82)$ & $1.28(1.11-1.48)$ & 0.001 \\
\hline \multicolumn{2}{|l|}{$\mathrm{LDH}(\mathrm{U} / \mathrm{L})$} & $229(185,292)$ & $1.01(1.01-1.02)$ & 0.001 \\
\hline \multicolumn{2}{|l|}{ D-dimer $(\mu \mathrm{g} / \mathrm{nL})(N=73)$} & $0.8(0.60,1.09)$ & $2.00(0.97-4.12)$ & 0.059 \\
\hline \multicolumn{2}{|l|}{$\mathrm{BNP}(\mathrm{pg} / \mathrm{mL})(N=85)$} & $8.6(3.1,21.7)$ & $1.01(0.998-1.03)$ & 0.098 \\
\hline \multicolumn{5}{|l|}{ Baseline CT results } \\
\hline \multicolumn{2}{|l|}{ Maximum subsegmental vascular diameter (mm) } & $4.75(4.15 .3)$ & $3.25(1.63-6.49)$ & $0.001 *$ \\
\hline \multirow[t]{5}{*}{ CT lesion extent } & Normal (\%) & $87.1(74.4,95.5)$ & $0.934(0.90-0.97)$ & $<0.001$ \\
\hline & GGO $(\%)$ & $4.2(1.1,12.0)$ & $1.08(1.02-1.15)$ & 0.013 \\
\hline & Reticulation (\%) & $2.1(0.5,6.6)$ & $1.20(1.08-1.35)$ & 0.001 \\
\hline & Consolidation $(\%)$ & $1.9(0.9,3.0)$ & $1.51(1.17-1.95)$ & 0.002 \\
\hline & Sum of lesion extent (\%) & $10.3(3.1,23.2)$ & $1.08(1.04-1.12)$ & $<0.001 *$ \\
\hline \multicolumn{2}{|l|}{ Absolute total CT lung volume (ml) } & $4112(3594,4789)$ & $0.999(0.999-1.00)$ & 0.036 \\
\hline \multicolumn{2}{|l|}{ Absolute normal lung volume (ml) } & $3591(2819,4386)$ & $0.999(0.999-1.00)$ & 0.001 \\
\hline \multicolumn{2}{|l|}{ Absolute total CT lesion volume (ml) } & $447(133,946)$ & $\begin{array}{l}1.003 \\
(1.001-1.004)\end{array}$ & $<0.001$ \\
\hline
\end{tabular}

$B N P$ brain natriuretic peptide, $C I$ confidence interval, $C T$ computed tomography, $I Q R$ interquartile range, $L D H$ lactate dehydrogenase, $S p O_{2}$ oxygen saturation, $G G O$ ground-glass opacity

${ }^{*}$ These data were included in the multivariate analysis

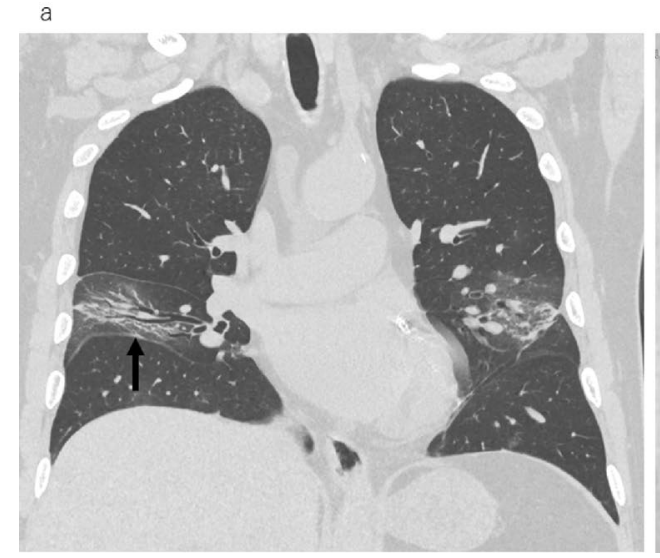

Fig. 2 Ultra-high-resolution computed tomography (CT) images from a 71-year-old male with fever on day 5 from symptom onset. a Coronal image showing a narrow space between the bronchi in the middle lobe and a shift of the right interlobar fissure toward the lesion

$p=0.013)$ for GGOs, $1.20(95 \%$ CI $1.08-1.35, p=0.001)$ for reticulation, and $1.51(95 \% \mathrm{CI} 1.17-1.95, p=0.002)$ for consolidation. A smaller CTLVtotal was associated with

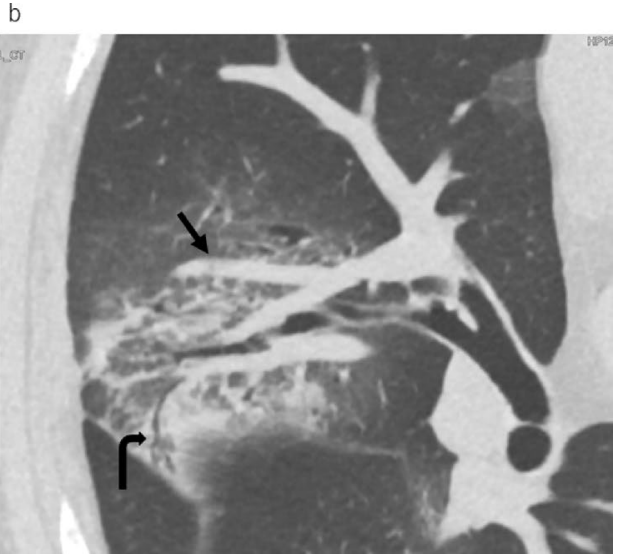

(arrow). b Magnified oblique axial image showing subsegmental vessel enlargement near the lesion (arrow). Peripheral bronchiolectasis is also seen (curved arrow). In this case, the sum of the CT lesion extent was $2.87 \%$

the severe-critical condition (odds ratio: $0.999 ; 95 \% \mathrm{CI}$ $0.999-1.00, p=0.036$ ). 

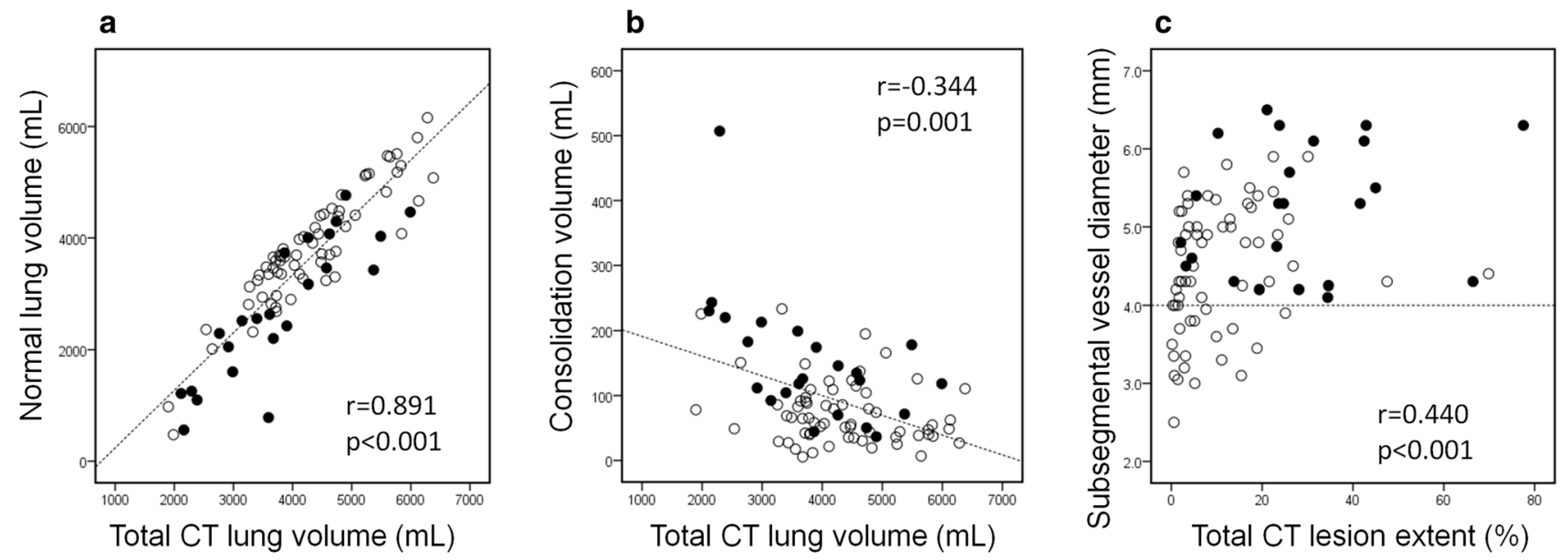

Fig. 3 Correlation between vascular diameter and computed tomography (CT) volume. White circles and black circles indicate patients with mild-moderate and severe-critical condition, respectively. a Scatter plot showing significant positive correlation between total CT lung volume and normal lung volume $(r=0.891, p<0.001)$. b Scatter plot showing weak negative correlation between total CT lung vol- ume and total consolidation volume $(r=-0.344, p=0.001)$. c Scatter plot showing correlation between maximum subsegmental vascular diameter and extent of CT lesions. A correlation was seen $(r=0.440$, $p<0.001)$, but vascular enlargement was seen in patients without a large amount of consolidation

Table 2 Comparison of subsegmental maximum vascular diameter, CT lesion extent, and laboratory data using Spearman's correlation coefficients

$$
\begin{array}{lllllll}
\mathrm{SpO}_{2}(N=87) & \mathrm{CRP}(N=87) & \mathrm{LDH}(N=87) & \mathrm{BNP}(N=83) & \mathrm{D} \text {-dimer }(N=72) & \begin{array}{l}
\text { Total CT lung } \\
\text { volume }(N=87)
\end{array} & \begin{array}{l}
\text { Vascular } \\
\text { diameter }
\end{array}
\end{array}
$$
$(N=87)$

\begin{tabular}{llllllll}
\hline Normal & 0.581 & -0.552 & -0.510 & -0.130 & -0.470 & 0.314 & -0.465 \\
Extent & $p<0.001$ & $p<0.001$ & $p<0.001$ & $p=0.242$ & $p<0.001$ & $p=0.003$ & $p<0.001$ \\
GGO & -0.419 & 0.433 & 0.483 & -0.012 & 0.273 & -0.332 & 0.355 \\
Extent & $p<0.001$ & $p<0.001$ & $p<0.001$ & $p=0.913$ & $p=0.017$ & $p=0.002$ & $p=0.001$ \\
Reticulation & -0.583 & 0.655 & 0.657 & 0.211 & 0.498 & -0.293 & 0.475 \\
Extent & $p<0.001$ & $p<0.001$ & $p<0.001$ & $p=0.056$ & $p<0.001$ & $p=0.006$ & $p<0.001$ \\
Consolidation & -0.505 & 0.520 & 0.507 & 0.189 & 0.372 & -0.551 & 0.473 \\
Extent & $p<0.001$ & $p<0.001$ & $p<0.001$ & $p=0.088$ & $p=0.001$ & $p<0.001$ & $p<0.001$ \\
Total CT lung Volume & 0.138 & -0.011 & -0.032 & -0.102 & -0.09 & 1 & -0.046 \\
& $p=0.203$ & $p=0.919$ & $p=0.77$ & $p=0.361$ & $p=0.452$ & -0.046 \\
Vascular Diameter & -0.406 & 0.484 & 0.266 & -0.092 & 0.320 & -0.673 \\
& $p<0.001$ & $p<0.001$ & $p=0.013$ & $p=0.407$ & $p=0.009$ & $p=0.673$ & 1 \\
\hline
\end{tabular}

$B N P$ brain natriuretic peptide, $C R P$ C-reactive protein, $C T$ computed tomography, $L D H$ lactate dehydrogenase, $\mathrm{SpO} \mathrm{O}_{2}$ oxygen saturation, $G G O$ ground-glass opacity

We performed multivariate logistic analysis of significant CT results and clinical factors. Since CT lesion extents of GGOs, reticulation, or consolidation per patient were mutually correlated, we adopted the sum of CT extent as a representative parameter of CT lesions [13]. Multivariate logistic analysis, including age, presence of comorbidity subsegmental vessel diameters, and CT lesion extent, showed subsegmental vessel diameter (odds ratio 3.05, 95\% CI 1.21-7.70, $p=0.018$ ), age (odds ratio 1.07, 95\% CI 1.01-1.13, $p=0.017$ ), and lesion extent on CT (odds ratio $1.07,95 \% \mathrm{CI} 1.02-1.11, p=0.002$ ) to be independent
Table 3 Multivariate logistic regression analysis for prognosing disease severity

\begin{tabular}{lllll}
\hline & Odds ratio & $\begin{array}{l}\text { 95\% confidence } \\
\text { interval }\end{array}$ \\
\hline Age & 1.07 & 1.012 & 1.132 & 0.017 \\
Presence of comorbidity & 1.637 & 0.39 & 3.875 & 0.501 \\
Sum of CT lesion extent & 1.066 & 1.024 & 1.110 & 0.002 \\
Pulmonary vessel diameter & 3.052 & 1.21 & 7.697 & 0.018 \\
\hline
\end{tabular}

predictors for disease severity. The presence of comorbidity was not significant $(p=0.501)$ (Table 3$)$. 
Figure 4 shows a comparison of the initial and followup CT results. The median interval between CT examinations was 51 days (range 30-96 days). Thirteen patients were treated with favipiravir (compassionate use). Corticosteroids were also used in twelve patients. Two patients were treated with remdesivir. Maximum subsegmental diameter $(p=0.001)$ and absolute CT lesion volume (sum of GGO, reticulation, and consolidation) $(p=0.002)$ significantly decreased on follow-up CT scans. Interestingly, CTLVtotal did not show a significant change (Fig. 4). In 4 of the 16 patients with the severe-critical condition, GGO and reticulation remained on the follow-up CT scans (Fig. 5). a CT lesion volume $(\mathrm{mL})$

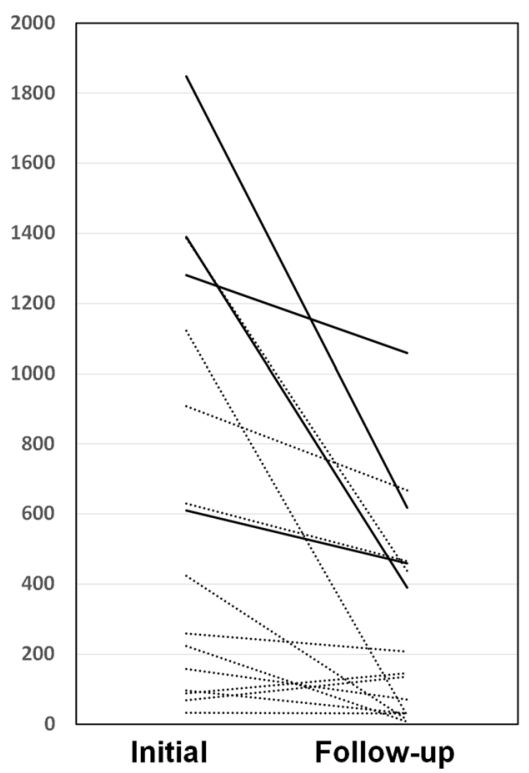

b Maximum Subsegmental vessel diameter $(\mathrm{mm})$

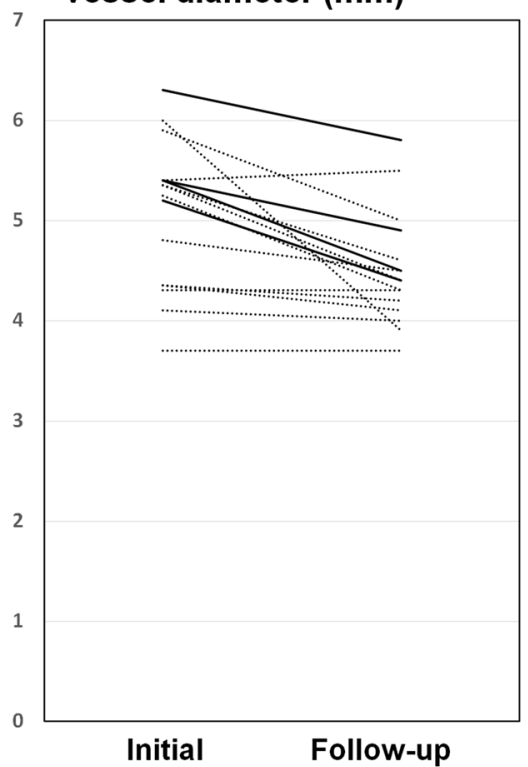

Total CT lung volume $(\mathrm{mL})$

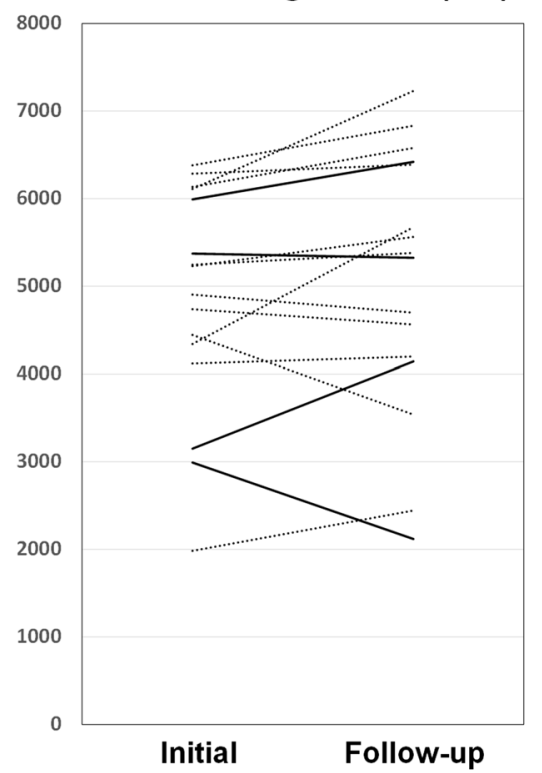

Fig. 4 Change in volume and subsegmental vessel diameter between initial and follow-up computed tomography (CT). Continuous lines indicate patients with severe-critical condition and dashed lines indicate patients with mild-moderate condition. a Scatter plot showing change in CT lesion volume (sum of GGO, reticulation, and consolidation). Lesions decreased on follow-up CT images $(p=0.005)$, but remained in patients with severe-critical condition. b Scatter plot showing change in maximum subsegmental vascular diameter, which decreased on follow-up CT images $(p=0.001)$. c Scatter plot showing change in total CT lung volume. There was no significant difference between timepoints
Fig. 5 Ultra-high-resolution computed tomography (CT) images of an 83-year-old female with cough and fatigue on a day 8 from symptom onset and b day 43 from symptom onset after treatment with favipiravir and corticosteroids. a Right lung showing ground-glass opacities and reticulation. The vessels close to the lesion are thicker than those on follow-up CT images (large arrows). b Reticulation with peripheral bronchiectasis (thin arrows) on follow-up CT. Total CT lung volume decreased from 2987 to $2117 \mathrm{ml}$ a

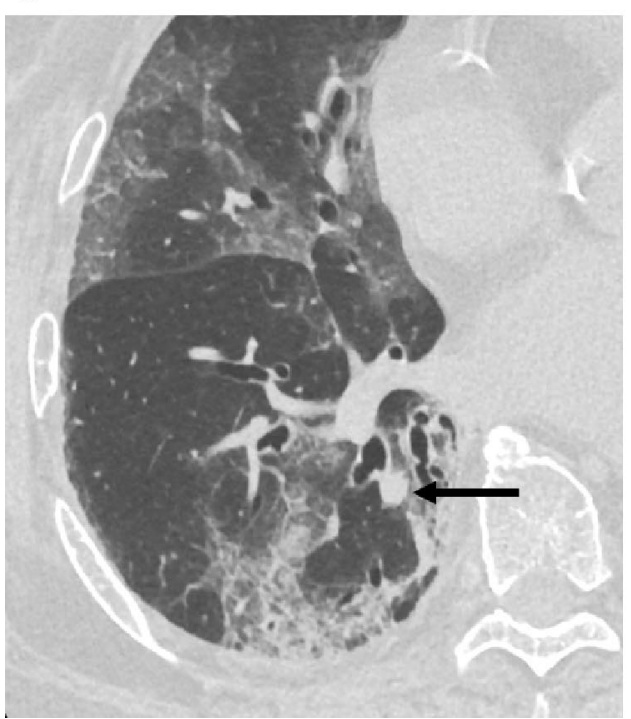

b

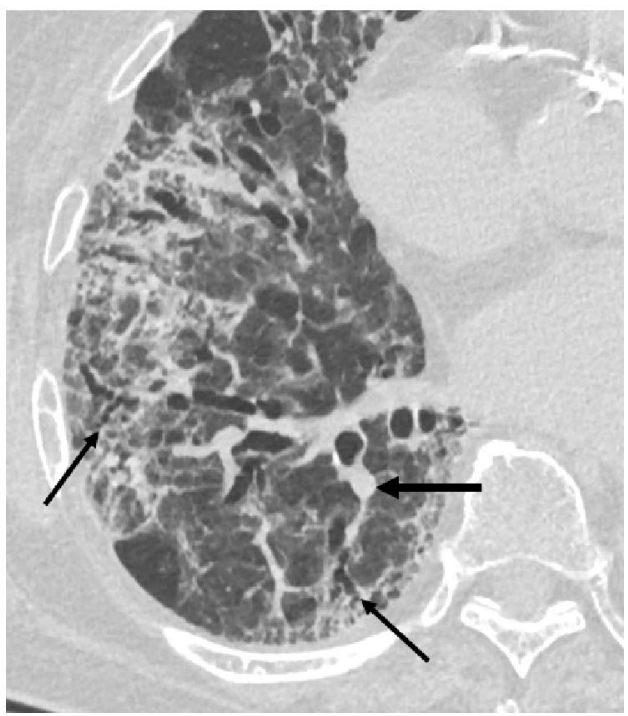




\section{Discussion}

In this study, we quantitatively measured the maximum subsegmental vascular diameter in the lung, CTLVtotal, and CT lesion extent (\%) in patients with COVID-19. Pulmonary vascular enlargement (maximum subsegmental diameter $>4 \mathrm{~mm}$ ) was seen in 66 patients $(75.9 \%)$ and in all patients with the severe-critical condition. Maximum subsegmental diameter was positively correlated with CT lesion extent measured using software. These results are compatible with the previous reports $[9,10]$. Moreover, multivariate logistic analysis showed that subsegmental vessel diameter was an independent predictor for disease severity (odds ratio $3.05, p=0.018$ ), as were age and lesion extent on CT. Thus, we consider subsegmental vascular enlargement to be a notable CT feature in COVID-19, especially in the acute phase.

In our results, the maximum subsegmental vascular diameter was positively correlated with CRP and D-dimer levels, but there was no significant correlation with BNP level. Our findings suggest that vascular enlargement is not reflected by cardiac failure, but that it would be correlated with inflammation and vascular impairment induced by SARS-CoV-2. Patel et al. found coagulation abnormalities in intubated patients with COVID-19, and at the same time, they showed that dilated peripheral vessels and perfusion defects were frequently seen on dual-energy CT [6]. These data suggest that vascular enlargement is not simply increased blood flow, but a manifestation of pulmonary thrombotic angiopathy due to COVID-19 [14-16].

Decreased CTLVtotal in patients with severe-critical condition in univariate analysis has already been reported [17-19]. We also found that CTLVtotal was negatively correlated with absolute consolidation volume. A previous experimental study reported interferon-mediated inflammatory responses, loss of surfactant proteins, and apoptosis of pneumocytes in infected alveolospheres [20]. Histological studies have reported diffuse alveolar damage in patients with ARDS caused by SARS-CoV-2 [21-23]. Lung volume loss due to alveolar collapse is a common phenomenon in ARDS [24]. Therefore, the volume loss of lesions may be related to the severity of lung injury, such as in the case of diffuse alveolar damage observed in ARDS. In other words, some patients might appear to have smaller CT lesions due to severe local volume loss. In these cases, vascular enlargement will be an important sign indicating disease severity.

In the follow-up study, we found that CTLVtotal did not increase and reticulation remained, especially in patients with a severe condition, which indicates residual fibrosis [25]. Recently, COVID-19 sequelae have been reported in the form of fatigue and dyspnea lasting beyond 60 days after onset [26]. As such, continued CT monitoring after discharge for longlasting effects is warranted.
The present study has several limitations. This was a retrospective, single-center study comprising a small number of patients. The laboratory data were limited, and blood gas was not measured in most cases. As a result, we could evaluate only four factors in the multivariate analysis, and the laboratory data were not adequately evaluated. Further studies with a larger number of subjects are required to confirm our findings. In this study, we manually measured vessel diameters according to a technique used in a previous study [9]. We assume that most of the vessel diameters measured were of pulmonary arteries; however, it is difficult to completely exclude pulmonary veins on $\mathrm{CT}$ images without using contrast materials. A previous report indicated that both pulmonary arteries and veins might enlarge due to disrupted vasoregulation in COVID-19 pneumonia [27]. We believe that UHR-CT, with eight times the spatial resolution of conventional CT, helped us to measure small vessel diameters precisely, as with peripheral bronchial diameters [28]. We consider the comparison of mean and maximum vessel diameters to be a simple, sensitive method to detect vascular enlargement. However, this method showed low specificity. Recently, automated measurement of whole-lung vessel volume has also been applied in patients with COVID-19 [29]. These computer-aided vessel measurements might provide more information about lung perfusion in non-contrast-enhanced CT.

In conclusion, subsegmental vessel enlargement was frequently observed in patients with COVID-19, especially those in severe and critical conditions. Enlargement of the subsegmental vessels is a notable finding related to circulatory impairments in COVID-19. Quantitative assessments, CT lesion volume, and vascular diameter will be useful for understanding the pathophysiological manifestations and predicting the disease severity of COVID-19.

\section{Compliance with ethical standards}

Conflict of interest Tae Iwasawa was provided software (Quantification by Ziosoft Informatics Platform for interstitial lung disease, QZIP) by Ziosoft (Tokyo, Japan). Tae Iwasawa received a research grant from Canon Medical Systems.

Ethics statement Our institutional review board approved of this retrospective, single-center study and waived the requirement for obtaining informed patient consent (KCRC-20-0008).

\section{References}

1. World Health Organization, Weekly epidemiological update-17 November 2020. https://www.who.int/publications/m/item/weekl y-epidemiological-update---17-november-2020. Accessed 22 Nov 2020.

2. Huang C, Wang Y, Li X, Ren L, Zhao J, Hu Y, et al. Clinical features of patients infected with 2019 novel coronavirus in Wuhan, China. Lancet. 2020;395:497-506. 
3. Gattinoni L, Coppola S, Cressoni M, Busana M, Rossi S, Chiumello D. COVID-19 does not lead to a "typical" acute respiratory distress syndrome. Am J Respir Crit Care Med. 2020;201:1299-300.

4. Pfeifer M, Ewig S, Voshaar T, Randerath WJ, Bauer T, Geiseler J, et al. Position paper for the state-of-the-art application of respiratory support in patients with COVID-19. Pneumologie. 2020;74:337-57.

5. Lang M, Som A, Mendoza DP, Flores EJ, Reid N, Carey D, et al. Hypoxaemia related to COVID-19: vascular and perfusion abnormalities on dual-energy CT. Lancet Infect Dis. 2020;20:1365-6.

6. Patel BV, Arachchillage DJ, Ridge CA, Bianchi P, Doyle JF, Garfield B, et al. Pulmonary angiopathy in severe COVID-19: physiologic, imaging, and hematologic observations. Am J Respir Crit Care Med. 2020;202:690-9.

7. Eslami V, Abrishami A, Zarei E, Khalili N, Baharvand Z, Sanei-Taheri M. The association of CT-measured cardiac indices with lung involvement and clinical outcome in patients with COVID-19. Acad Radiol. 2020. https://doi.org/10.1016/j. acra.2020.09.012.

8. Spagnolo P, Cozzi A, Foà RA, Spinazzola A, Monfardini L, Bnà $\mathrm{C}$, et al. CT-derived pulmonary vascular metrics and clinical outcome in COVID-19 patients. Quant imaging med surg. 2020;10:1325-33.

9. Caruso D, Zerunian M, Polici M, Pucciarelli F, Polidori T, Rucci C, et al. Chest CT features of COVID-19 in Rome, Italy. Radiology. 2020. https://doi.org/10.1148/radiol.2020201237.

10. Wang C, Shi B, Wei C, Ding H, Gu J, Dong J. Initial CT features and dynamic evolution of early-stage patients with COVID-19. Radiol Infect Dis. 2020. https://doi.org/10.1016/j. jrid.2020.08.002.

11. Valentin J, International Commission on Radiation Protection. Managing patient dose in multi-detector computed tomography (MDCT). ICRP Publication 102. Ann ICRP. 2007;37:1-79.

12. National Institutes of Health. Coronavirus Disease 2019 (COVID19) Treatment Guidelines. https://www.covid19treatmentguid elines.nih.gov (accessed 22 Nov 2020).

13. Gönen M, Panageas KS, Larson SM. Statistical issues in analysis of diagnostic imaging experiments with multiple observations per patient. Radiology. 2001;221(3):763-7.

14. Ackermann M, Verleden SE, Kuehnel M, Haverich A, Welte T, Laenger F, et al. Pulmonary vascular endothelialitis, thrombosis, and angiogenesis in Covid-19. N Engl J Med. 2020;383:120-8.

15. Huertas A, Montani D, Savale L, Pichon J, Tu L, Parent F, et al. Endothelial cell dysfunction: a major player in SARS-CoV-2 infection (COVID-19)? Eur Respir J. 2020;56:2001634.

16. Cavagna E, Muratore F, Ferrari F. Pulmonary thromboembolism in COVID-19: venous thromboembolism or arterial thrombosis? Radiology. 2020. https://doi.org/10.1148/ryct.2020200289.

17. Fang X, Li X, Bian Y, Ji X, Lu J. Relationship between clinical types and radiological subgroups defined by latent class analysis in 2019 novel coronavirus pneumonia caused by SARS-CoV-2. Eur Radiol. 2020;2:1-12.

18. Colombi D, Bodini FC, Petrini M, Maffi G, Morelli N, Milanese $\mathrm{G}$, et al. Well-aerated lung on admitting chest CT to predict adverse outcome in COVID-19 pneumonia. Radiology. 2020;296:e86-96.

19. Iwasawa T, Sato M, Yamaya T, Sato Y, Uchida Y, Kitamura H, et al. Ultra-high-resolution computed tomography can demonstrate alveolar collapse in novel coronavirus (COVID-19) pneumonia. Jpn J Radiol. 2020;38(5):394-8.

20. Katsura H, Sontake V, Tata A, Kobayashi Y, Edwards CE, Konkimalla A, et al. Human lung stem cell-based alveolospheres provide insights into SARS-CoV-2-mediated interferon responses and pneumocyte dysfunction. Cell Stem Cell. 2020;27:890-904.

21. Barton LM, Duval EJ, Stroberg E, Ghosh S, Mukhopadhyay S. COVID-19 autopsies, Oklahoma, USA. Am J Clin Pathol. 2020;153:725-33.

22. Tian S, Hu W, Niu L, Liu H, Xu H, Xiao SY. Pulmonary pathology of early-phase 2019 novel coronavirus (COVID-19) pneumonia in two patients with lung cancer. J Thorac Oncol. 2020;15:700-4.

23. Zhang H, Zhou P, Wei Y, Yue H, Wang Y, Hu M, et al. Histopathologic changes and SARS-CoV-2 immunostaining in the lung of a patient with COVID-19. Ann intern med. 2020. https://doi. org/10.7326/M20-0533.

24. Puybasset L, Cluzel P, Chao N, Slutsky AS, Coriat P, Rouby JJ. A computed tomography scan assessment of regional lung volume in acute lung injury. The CT Scan ARDS Study Group. Am J Respir Crit Care Med. 1998;158:1644-55.

25. Ojha V, Mani A, Pandey NN, Sharma S, Kumar S. CT in coronavirus disease 2019 (COVID-19): a systematic review of chest CT findings in 4410 adult patients. Eur Radiol. 2020;30:6129-38.

26. Carfì A, Bernabei R, Landi F. Gemelli against COVID-19 postacute care study group. Persistent symptoms in patients after acute COVID-19. JAMA. 2020;324:603-5.

27. Herrmann J, Mori V, Bates JHT, Suki B. Modeling lung perfusion abnormalities to explain early COVID-19 hypoxemia. Nat Commun. 2020;11:4883.

28. Morita Y, Yamashiro T, Tsuchiya N, Tsubakimoto M, Murayama S. Automatic bronchial segmentation on ultra-HRCT scans: advantage of the 1024-matrix size with 0.25 -mm slice thickness reconstruction. Jpn J Radiol. 2020;38:953-9.

29. Thillai M, Patvardhan C, Swietlik EM, McLellan T, De Backer J, Lanclus $\mathrm{M}$, et al. Functional respiratory imaging identifies redistribution of pulmonary blood flow in patients with COVID-19. Thorax. 2020. https://doi.org/10.1136/thoraxjnl-2020-215395.

Publisher's Note Springer Nature remains neutral with regard to jurisdictional claims in published maps and institutional affiliations. 